\title{
A METHOD OF SOLVING A SYSTEM OF LINEAR EQUATIONS WHOSE COEFFICIENTS FORM A TRIDIAGONAL MATRIX*
}

\author{
BY \\ THOMAS C. T. TING \\ Brown University
}

1. Introduction. Consider the following system of simultaneous linear equations

$$
\begin{aligned}
-2 x_{1}+x_{2} & =d_{1}, \\
x_{1}-2 x_{2}+x_{3} & =d_{2}, \\
x_{2}-2 x_{3}+x_{4} & =d_{3}, \\
x_{3}-2 x_{4}+x_{5} & =d_{4}, \\
x_{4}-x_{5} & =d_{5},
\end{aligned}
$$

where $d_{i}(i=1,2, \cdots, 5)$ are known quantities. Suppose we want to solve Eqs. (1) for different sets of $d_{i}$. To be more general, suppose we want to solve the system of equations

$$
A \mathbf{x}=\mathrm{d},
$$

where $A$ is a non-singular tridiagonal matrix (not necessarily symmetric) whose elements are given and fixed while $\mathrm{d}$ will be changed from time to time. Since $A$ is nonsingular, one can obtain the inverse $A^{-1}$ of $A$ and determine $\mathbf{x}$ by

$$
\mathbf{x}=A^{-1} \mathrm{~d} \text {. }
$$

$A^{-1}$ so obtained, however, is no longer a tridiagonal matrix. If $A$ is a $n \times n$ matrix, we will have in general $n^{2}$ elements of $A^{-1}$. When $n$ is large, this method becomes unwieldy, even with an electronic computer.

Linear equations similar to Eqs. (1) are frequently encountered in problems of mathematical physics. For instance, the backward finite difference method for solving the heat equation requires the solution of equations (2) for each step where $A$ is fixed and $d$ is changed from step to step. Heat equations also appear in the study of longitudinal impact on visco-plastic rods. The solution of other problems, such as discretely loaded strings and the application of the three moment theorem to continuous beams, result in equations of the form of (2).

Methods are known which enable one to determine $\mathbf{x}$ of (2) more efficiently than by using Eq. (3) (see [1], [2] for example). In the following, we will present another method which is very efficient and convenient for ordinary physical problems. The comparison with the traditional triangular decomposition method is presented in the Appendix.

${ }^{*}$ Received June 19, 1963. The work presented here was supported by the National Science Foundation through Grant GP1115. 
2. The method. We shall discuss the following system of equations:

$$
\begin{aligned}
b_{1} x_{1}+a_{1}^{\prime} x_{2} & =d_{1}, \\
a_{2} x_{1}+b_{2} x_{2}+a_{2}^{\prime} x_{3} & =d_{2}, \\
a_{3} x_{2}+b_{3} x_{3}+a_{3}^{\prime} x_{4} \ldots \ldots \ldots \ldots \ldots \ldots & =d_{3}, \\
\ldots \ldots \ldots \ldots \ldots \ldots \ldots \ldots \ldots & \cdots \cdots \\
a_{n-1} x_{n-2}+b_{n-1} x_{n-1}+a_{n-1}^{\prime} x_{n} & =d_{n-1}, \\
a_{n} x_{n-1}+b_{n} x_{n} & =d_{n} .
\end{aligned}
$$

To discuss our method, we will consider first a special case in which $d_{1}=d_{2}=\cdots=$ $d_{n-1}=0$ but $d_{n} \neq 0$. We define:

$$
c_{2}=-a_{1}^{\prime} / b_{1}, \quad c_{i+1}=-a_{i}^{\prime} /\left(a_{i} c_{i}+b_{i}\right) .
$$

Starting from the first equation of (4) by Gaussian eliminations, we obtain:

$$
\begin{aligned}
x_{1} & =c_{2} x_{2}, \\
x_{2} & =c_{3} x_{3}, \\
\cdots & \cdots \cdots, \\
x_{n-1} & =c_{n} x_{n}, \\
x_{n} & =d_{n} /\left(a_{n} c_{n}+b_{n}\right) .
\end{aligned}
$$

\begin{tabular}{|c|c|c|c|c|c|}
\hline \multicolumn{6}{|c|}{$d_{1}=d_{2}=d_{3}=d_{4}=0, d_{5}=-1$, Eqs. } \\
\hline (1) & $i=1$ & 2 & 3 & 4 & 5 \\
\hline (2) & $c_{i}=$ & $1 / 2$ & $2 / 3$ & $3 / 4$ & $4 / 5$ \\
\hline (3) & $\overline{x_{i}=1}$ & -2 & - & $\leftarrow 4$ & -5 \\
\hline
\end{tabular}

Hence we determine $x_{n}$ from the last equation of (6) and then determine the other values $x_{n-1}, x_{n-2}, \cdots, x_{1}$.

Although this is trivial, let us illustrate this special case by taking $d_{1}=d_{2}=d_{3}=d_{4}=0$, $d_{5}=-1$ in Eqs. (1). The values of $c_{i}$ for Eqs. (1) are listed in the second row of Table 1. The last row of Table 1 gives the solution for $x_{i}$. Notice that $x_{5}=5$ by the last equation of (6). The other equations of (6) give $x_{4}=c_{5} x_{5}=4, x_{3}=c_{4} x_{4}=3$ and so on. Hence $c_{i}$ is the "left carry-over factor" of $x_{i}$ to $x_{i-1}$.

TABLe 1

In the same manner, one can introduce the "right carry-over factors". Indeed, when $d_{1} \neq 0$ and $d_{2}=d_{3}=\cdots=d_{n}=0$, one obtains

$$
\begin{aligned}
x_{n} & =c_{n-1}^{\prime} x_{n-1}, \\
x_{n-1} & =c_{n-2}^{\prime} x_{n-2} \\
\cdots \cdots \cdots \cdots \cdots, & \cdots \cdots, \\
x_{2} & =c_{1}^{\prime} x_{1}, \\
x_{1} & =d_{1} /\left(a_{1}^{\prime} c_{1}^{\prime}+b_{1}\right),
\end{aligned}
$$


where

$$
c_{n-1}^{\prime}=-a_{n} / b_{n}, \quad c_{i-1}^{\prime}=-a_{i} /\left(a_{i}^{\prime} c_{i}^{\prime}+b_{i}\right) .
$$

Taking Eqs. (1) as an example again, we put $d_{1}=-1, d_{2}=d_{3}=d_{4}=d_{5}=0$. The solution for $x_{i}$ is shown in Table 2 .

TABLE 2

\begin{tabular}{|c|c|c|c|c|c|}
\hline \multicolumn{6}{|c|}{$d_{1}=-1, d_{2}=d_{3}=d_{4}=d_{5}=0$, Eqs. (1) } \\
\hline (1) & $i=1$ & 2 & 3 & 4 & 5 \\
\hline (2) & $c_{1}^{\prime}=1$ & 1 & 1 & 1 & 1 \\
\hline (3) & $x_{i}=1$ & & 1 & & 1 \\
\hline
\end{tabular}

Next, we consider the case when $d_{i}$ are all zero except $d_{k}$ where $0<k<n$. The first $(k-1)$ equations of $(4)$ give:

$$
\begin{gathered}
x_{1}=c_{2} x_{2}, \\
x_{2}=c_{3} x_{3}, \\
\cdots \cdots \cdots \\
x_{k-1}=c_{k} x_{k} .
\end{gathered}
$$

The last $(n-k)$ equations of (4) give:

$$
\begin{aligned}
x_{n} & =c_{n-1}^{\prime} x_{n-1}, \\
x_{n-1} & =c_{n-2}^{\prime} x_{n-2}, \\
\cdots \cdots & \cdots \cdots \cdots, \\
x_{k+1} & =c_{k}^{\prime} x_{k} .
\end{aligned}
$$

Hence the $k$ th equation of (4) gives:

$$
a_{k} c_{k} x_{k}+b_{k} x_{k}+a_{k}^{\prime} c_{k}^{\prime} x_{k}=d_{k} .
$$

Let us define

$$
w_{i}=1 /\left(a_{i} c_{i}+b_{i}+a_{i}^{\prime} c_{i}^{\prime}\right)
$$

Then we have

$$
x_{k}=w_{k} d_{k}
$$

$w_{i}$ will be called the "weight factor" of $x_{i}$. As an illustration, let $d_{3}=-1, d_{1}=d_{2}=$ $d_{4}=d_{5}=0$ in Eqs. (1). The solution for this case is shown in Table 3. The right carryover factors $c_{i}^{\prime}$ are shown in the second row with the arrow heads $(\rightarrow)$ between the factors. Similarly, the left carry-over factors $c_{i}$ are listed in the fourth row with the arrow heads $(\leftarrow)$ between the factors. With these arrow heads, it is easier to identify which carry-over factor is to be used in the calculations. 
TABLE 3

$d_{1}=d_{2}=d_{4}=d_{5}=0, d_{3}=-1$, Eqs. (1)

$$
\begin{array}{rlrrrrr}
i=1 & & 2 & 3 & 4 & 5 \\
\hline c_{i}^{\prime}=1 & \rightarrow & 1 & \rightarrow & \rightarrow & 1 & \rightarrow \\
w_{i}= & \\
c_{i}= & \leftarrow 1 / 2 & \leftarrow 2 / 3 & \leftarrow 3 / 4 & \leftarrow 4 / 5 \\
\hline x_{i}=1 & \leftarrow 2 & \leftarrow 3 & \rightarrow & \rightarrow & \rightarrow & 3
\end{array}
$$

Finally, we will illustrate the general case in which $d_{i}$ are all specified. It is seen from the previous examples that the values of $x_{i}$ in general consist of three parts, namely: the value due to its own weight, that due to the right carry-over, and that due to the left carry-over. If we denote these values by $W_{i}, C_{i}^{\prime}$ and $C_{i}$ respectively, we have

$$
x_{i}=W_{i}+C_{i}^{\prime}+C_{i} \text {. }
$$

To illustrate this, we take $d_{1}=d_{2}=d_{3}=d_{4}=d_{5}=-1$ in Eqs. (1). The values $W_{i}$ are obtained by

$$
W_{i}=w_{i} d_{i} \text {, }
$$

where $w_{i}$ is the weight factor expressed by Eq. (9). Notice that $c_{1}$ and $c_{5}^{\prime}$ are taken as zero in obtaining $w_{1}$ and $w_{5}$. The values of $w_{i}$ and $W_{i}$ for this case are shown respectively in the third and sixth rows of Table 4 . The procedure of calculation may be followed in the table. For instance,

$$
\begin{array}{ll}
C_{2}^{\prime}=W_{1} c_{1}^{\prime}=1, & C_{3}^{\prime}=\left(W_{2}+C_{2}^{\prime}\right) c_{2}^{\prime}=(2+1) \cdot 1=3 ; \\
C_{4}=W_{5} c_{5}=4, & C_{2}=\left(W_{3}+C_{3}\right) c_{3}=(3+6) \cdot 2 / 3=6 .
\end{array}
$$

In other words, $C_{i}^{\prime}$ and $C_{i}$ are obtained by the recurrent formulae

$$
\begin{aligned}
& C_{i+1}^{\prime}=\left(W_{i}+C_{i}^{\prime}\right) c_{i}^{\prime} \\
& C_{i-1}=\left(W_{i}+C_{i}\right) c_{i} .
\end{aligned}
$$

It should be noticed that $C_{i}^{\prime}$ and $C_{i}$ are obtained independently. One can calculate $C_{i}^{\prime}$ first and then $C_{i}$ or vice versa. $W_{i}$, however, must be calculated before $C_{i}^{\prime}$ and $C_{i}$. Hence, the method has symmetry in the procedure of calculation.

If we are given another set of $d_{i}$ in Eqs. (1), we can use the same values of $c_{i}^{\prime}, c_{i}$ and $w_{i}$ in Table 4 to calculate $W_{i}, C_{i}^{\prime}$ and $C_{i} \cdot c_{i}^{\prime}, c_{i}$ and $w_{i}$ are obtained from $a_{i}^{\prime}, a_{i}$ and $b_{i}$. Once the former are obtained the latter are no longer needed. Notice that the former has exactly the same number of elements as the latter does.

It is seen from Tables 1,2 and 3 that if there is only one non-zero $d_{i}$ it is not necessary to calculate all of the carry-over factors and the weight factors to obtain the solution for $x_{i}$. On the other hand, if we want to know only one of the $x_{i}$, say $x_{k}$, we do not need to calculate all of the "carry-over values" $C_{i}^{\prime}$ and $C_{i}$. Indeed, only those $C_{i}^{\prime}$ and $C_{i}$ where $i \leq k, j \geq k$ are needed.

In deriving Eqs. (5), (8) and (9), we tacitly assumed that the denominators do not 
TABLE 4

$d_{1}=d_{2}=d_{3}=d_{4}=d_{5}=-1$, Eqs. (1)

(1)

$\begin{array}{rllllllll}i & =1 & 2 & 3 & & 4 & & 5 \\ c_{i}^{\prime}= & 1 & \rightarrow & 1 & \rightarrow & 1 & \rightarrow & 1 & \rightarrow\end{array}$

$w_{i}=-1 \quad-2 \quad-3 \quad-4 \quad-5$

$c_{i}=\quad \leftarrow 1 / 2 \leftarrow 2 / 3 \leftarrow 3 / 4 \leftarrow 4 / 5$

$W_{i}=1 \longrightarrow_{2}^{1} \longrightarrow_{3}^{3} \longrightarrow{ }_{0}^{3} \longrightarrow{ }_{0}^{6}$

7) $\quad C_{i}=4 \longleftarrow \bigcup_{6} \longleftarrow \bigcup_{6} \longleftarrow$

\begin{tabular}{llllll}
\hline$x_{i}=$ & 5 & 9 & 12 & 14 & 15
\end{tabular}

vanish. It can be shown that the denominator of Eq. (9) does not vanish if $A$ is nonsingular.

In Eq. (5) we can assume that $b_{1} \neq 0$. Should $b_{1}$ be zero, one can solve $x_{2}$ explicitly from Eqs. (4) and Eqs. (4) are reduced to similar equations with one unknown less. Similarly, we can assume that $b_{n} \neq 0$ in Eq. (8).

It remains to investigate the possibility that $\left(a_{i} c_{i}+b_{i}\right)$ of Eq. (5) and $\left(a_{i}^{\prime} c_{i}^{\prime}+b_{i}\right)$ of Eq. (8) may vanish, in other words $c_{i}$ and $c_{i}^{\prime}$ may be infinite. We will see later that $c_{k}=\infty$ implies that the value $x_{k}$ of Eqs. (4) does not depend on $d_{k}, d_{k+1}, \cdots, d_{n}$. Similarly, when $c_{k}^{\prime}=\infty, x_{k}$ does not depend on $d_{1}, d_{2}, \cdots, d_{k}$. In many physical problems, for instance in the solution of the heat equation by the backward difference method, the value of $x_{k}$ depends on all the values $d_{i}$. Hence, in most physical problems $c_{i}$ and $c_{i}^{\prime}$ rarely become infinite. For completeness of our method, however, we shall discuss this case in the next section.

3. Modified carry-over factors. As in the first example of section 2 , we assume that $d_{1}=d_{2}=\cdots=d_{n-1}=0, d_{n} \neq 0$ in Eqs. (4). By Gaussian elimination to the $(k-1)$ th equation, one obtains Eqs. (4) in the following form:

$$
\begin{gathered}
x_{1}=c_{2} x_{2}, \\
x_{2}=c_{3} x_{3}, \\
\cdots \cdots \cdots, \\
x_{k-2}=c_{k-1} x_{k-1}, \\
\left(a_{k-1} c_{k-1}+b_{k-1}\right) x_{k-1}+a_{k-1}^{\prime} x_{k}=0, \\
a_{k} x_{k-1}+b_{k} x_{k}+a_{k}^{\prime} x_{k+1}=0, \\
\cdots \cdots \cdots \cdots \cdots \cdots \cdots \cdots \cdots \cdots \cdots \\
a_{n} x_{n-1}+b_{n} x_{n}=d_{n} ;
\end{gathered}
$$

$c_{2}, c_{3}, \cdots, c_{k-1}$ are determined by (5) and we assume that they are all finite up to $c_{k-1}$. Suppose $\left(a_{k-1} c_{k-1}+b_{k-1}\right)=0$ so that $c_{k}$ does not exist. First we show that $\left(a_{k-1} c_{k-1}+b_{k-1}\right)$ and $a_{k}$ cannot both vanish if the matrix $A$ is non-singular. Indeed, if both vanish, the 
following set of $x_{i}$ will satisfy the corresponding homogeneous equations of (10):

$$
\begin{gathered}
x_{1}=c_{2} x_{2}, \quad x_{2}=c_{3} x_{3}, \cdots, x_{k-2}=c_{k-1} x_{k-1}, \\
x_{k}=x_{k+1}=\cdots=x_{n}=0 .
\end{gathered}
$$

By assuming an arbitrary non-zero value for $x_{k-1}$ in (11) one gets a non-trivial solution of Eqs. (10) with $d_{n}=0$. Since Eqs. (10) and Eqs. (4) are equivalent, this implies that Eqs. (4) has a non-trivial solution when $d_{i}$ are zero. This contradicts the assumption that the matrix $A$ is non-singular. Hence $\left(a_{k-1} c_{k-1}+b_{k-1}\right)$ and $a_{k}$ cannot both vanish.

When $\left(a_{k-1} c_{k-1}+b_{k-1}\right)=0$, Eqs. (10) give

We define:

$$
x_{k}=0, \quad x_{k-1}=-\left(a_{k}^{\prime} / a_{k}\right) x_{k+1} .
$$

$$
c_{k+1}=0, \quad c_{(k+1)}=-a_{k}^{\prime} / a_{k} \text { when } c_{k}=\infty .
$$

Then we have

$$
x_{k}=c_{k+1} x_{k+1}=0, \quad x_{k-1}=c_{(k+1)} x_{k+1} .
$$

$c_{(k+1)}$ will be called the "modified left carry-over factor" of $x_{k+1}$. It defines the portion of $x_{k+1}$ to be carried over to $x_{k-1}$ instead of $x_{k}$. Since we have proved that $a_{k} \neq 0, c_{(k+1)}$ exists.

The procedure of deriving the modified carry-over factors is equivalent to interchanging two equations to avoid a zero pivot.

It should be noticed from Eqs. (10) that when $\left(a_{k-1} c_{k-1}+b_{k-1}\right)=0$, i.e., $c_{k}=\infty, x_{k}$ is zero even if $d_{k}, d_{k+1}, \cdots, d_{n}$ are non-zero. Hence $x_{k}$ is independent of $d_{k}, d_{k+1}, \cdots, d_{n}$ when $c_{k}=\infty$.

By decomposing $A$ into a lower triangular matrix and an unit upper triangular matrix (see Eq. (22) of the Appendix), one can show that $\left(a_{k-1} c_{k-1}+b_{k-1}\right)=0$ implies that the $(k-1)$ th leading principal minor of $A$,

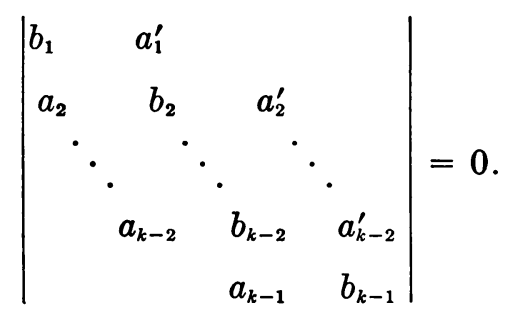

Hence, $\left(a_{i} c_{i}+b_{i}\right)=0$ if and only if a leading principal minor of $A$ vanishes. If $A$ is symmetric positive definite (which is the case in many physical problems), no minors are zero [3].

As an example, consider the following system of equations:

$$
\begin{aligned}
-2 x_{1}+x_{2} & =d_{1}, \\
2 x_{1}-x_{2}+x_{3} & =d_{2}, \\
x_{2}-2 x_{3}+x_{4} & =d_{3}, \\
x_{3}-2 x_{4}+x_{5} & =d_{4}, \\
x_{4}-x_{5} & =d_{5} .
\end{aligned}
$$


The left carry-over factors $c_{i}$ are listed in the fourth row of Table 5 . Here we have $c_{2}=\frac{1}{2}$ and $c_{3}=\infty$. Hence $c_{4}=0$ and $c_{(4)}=-1$ by our definition. In Table 5 , we do not list $c_{3}$ and $c_{4}$. In the place of $c_{4}$, we put $c_{(4)}$. The long arrow head between $c_{2}$ and $c_{(4)}$ suggests that the carry-over from $i=4$ should skip $i=3$ and go to $i=2$. There is no carry-over to $i=3$. In the fifth row we give a special case in which $d_{1}=d_{2}=d_{3}=$ $d_{4}=0, d_{5}=-2$. The solution for this case is shown in the sixth row. $x_{3}=0$ in this case.

A similar definition can be applied to the modified right carry-over factors. We define: when $c_{k}^{\prime}=\infty, c_{k-1}^{\prime}=0$ and $x_{k+1}=c_{(k-1)}^{\prime} x_{k-1}$ where $c_{(k-1)}^{\prime}=-a_{k} / a_{k}^{\prime}$. The right carry-over factors $c_{i}^{\prime}$ for Eqs. (12) are shown in the second row of Table 5. Here we have $c_{1}^{\prime}=\infty$. Since there is no $c_{0}^{\prime}$, we can ignore the modified carry-over factor $c_{(0)}^{\prime}$.

Table 5. Solutions of Eqs. (12)

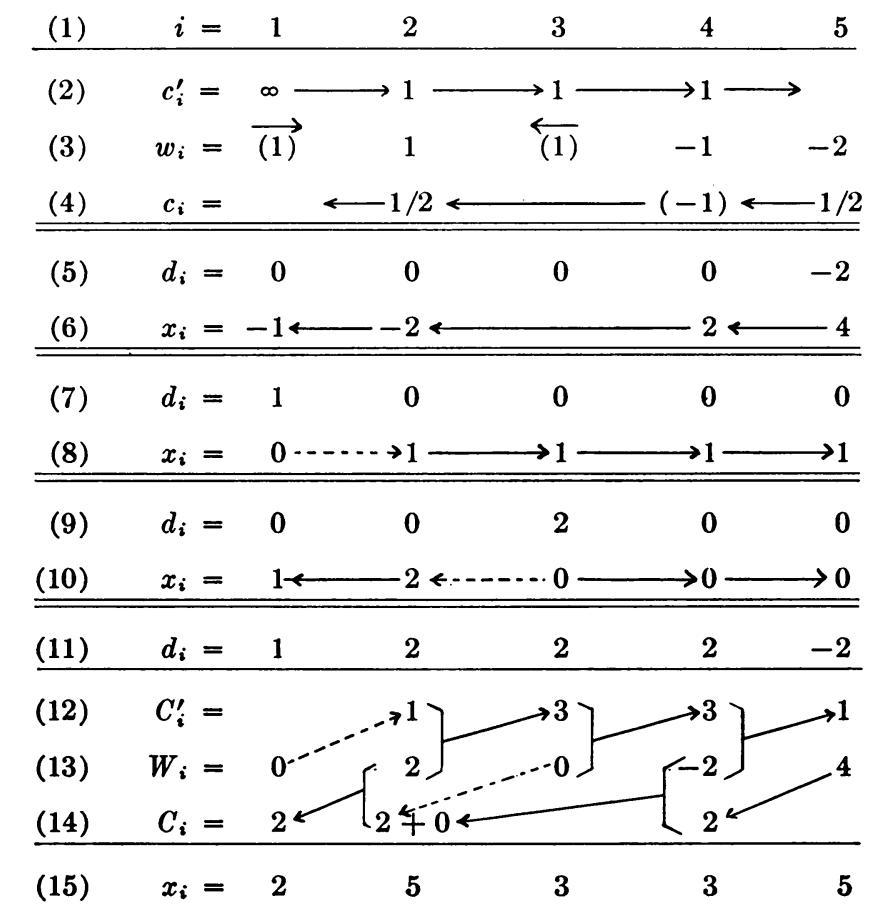

Finally, we consider the case in which $d_{i}$ are all zero except $d_{k}$, where $0<k<n$. By Gaussian eliminations from the first and the last of Eqs. (4) respectively, the $(k-1)$ th, $k$ th and $(k+1)$ th equations of $(4)$ can be written as:

$$
\begin{aligned}
\left(a_{k-1} c_{k-1}+b_{k-1}\right) x_{k-1}+a_{k-1}^{\prime} x_{k} & =0, \\
a_{k} x_{k-1}+b_{k} x_{k}+a_{k}^{\prime} x_{k+1} & =d_{k}, \\
a_{k+1} x_{k}+\left(b_{k+1}+a_{k+1}^{\prime} c_{k+1}^{\prime}\right) x_{k+1} & =0 .
\end{aligned}
$$

If $c_{k}, c_{k}^{\prime}$ both exist, the second equation of (13) yields $x_{k}=w_{k} d_{k}$ as discussed in Eq. (9). We shall investigate the case in which $c_{k}$ or $c_{k}^{\prime}$ is infinite, i.e., the case in which $\left(a_{k-1} c_{k-1}+b_{k-1}\right)$ or $\left(b_{k+1}+a_{k+1}^{\prime} c_{k+1}^{\prime}\right)$ vanishes.

First, one can prove that $\left(a_{k-1} c_{k-1}+b_{k-1}\right)$ and $\left(b_{k+1}+a_{k+1}^{\prime} c_{k+1}^{\prime}\right)$ cannot both vanish if $A$ is non-singular. The proof is similar to the one we proved in the beginning of this 
section. Should they both vanish, one can find a non-trivial solution of Eqs. (13) with $d_{k}=0$ and hence a non-trivial solution of Eqs. (4) with all $d_{i}=0$. This leads to a contradiction.

Suppose $\left(a_{k-1} c_{k-1}+b_{k-1}\right)=0$ while $\left(b_{k+1}+a_{k+1}^{\prime} c_{k+1}^{\prime}\right) \neq 0$. Eqs. (13) give:

$$
x_{k}=0, \quad x_{k+1}=0, \text { and } x_{k-1}=d_{k} / a_{k} .
$$

We define:

When $c_{k}=\infty, w_{k}=0$ and $x_{k-1}=w_{(k)} d_{k}$ where $w_{(k)}=1 / a_{k} . w_{(k)}$ is the modified weight factor which defines the weight factor by which $d_{k}$ should be "carried-over" to $x_{k-1}$ instead of "imposed" on $x_{k}$. The fact that $\left(a_{k-1} c_{k-1}+b_{k-1}\right)$ and $a_{k}$ cannot both vanish assures us the existence of $w_{(k)}$.

To illustrate the use of $w_{(k)}$, we take $d_{1}=d_{2}=d_{4}=d_{5}=0, d_{3}=2$ in Eqs. (12). This is shown in the ninth row of Table 5. Since $c_{3}=\infty, w_{3}=0$ and $w_{(3)}=1$ by our definition. In the third row, in the place of $w_{3}$, we put $w_{(3)}$. The arrow head on the top of $w_{(3)}$ suggests that the "weight" of $d_{3}$ should be carried over to $x_{2}$ which is to the "left" of $x_{3}$. Now, since $w_{3}=0, x_{3}=w_{3} d_{3}=0$ and $x_{2}=w_{(3)} d_{3}=2 . x_{4}=c_{3}^{\prime} x_{3}=0, x_{5}=$ $c_{4}^{\prime} x_{4}=0$ and $x_{1}=c_{2} x_{2}=1$. This is shown in the tenth row.

The counterpart of $w_{(k)}$ is $w_{(k)}^{\prime}$, which is defined in the same manner:

$$
\text { When } c_{k}^{\prime}=\infty, w_{k}=0 \text { and } x_{k+1}=w_{(k)}^{\prime} d_{k} \text { where } w_{(k)}^{\prime}=1 / a_{k}^{\prime} \text {. }
$$

In Eqs. (12), $c_{1}^{\prime}=\infty$. Hence $w_{1}=0$ and $w_{(1)}^{\prime}=1$. This is shown in the third row of Table 5. The solution of Eqs. (12) when $d_{i}$ are specified in the seventh row is shown in the eighth row.

In closing this section we give an example in which $d_{i}$ of Eqs. (12) are all specified as indicated in the eleventh row of Table 5 . The procedure of calculation is the same as in Table 4 except that whenever $w_{k}=0$, the values $w_{(k)} d_{k}$ or $w_{(k)}^{\prime} d_{k}$ are not placed in the row of $W_{i}$ but carried over to the row $C_{i}$ or $C_{i}^{\prime} \cdot w_{(k)} d_{k}$ and $w_{(k)}^{\prime} d_{k}$ are easily identified in Table 5 by the dotted-lined arrow heads.

4. An application. The method is also applicable to problems such as that of heat conduction in a semi-infinite rod, where the difference equations involve an "infinite" number of dependent variables. To illustrate, we consider the problem represented as follows:

$$
\begin{array}{rlrl}
\frac{\partial^{2} v}{\partial x^{2}}-\frac{\partial v}{\partial t} & =0, & \\
v(x, 0) & =0, & & 0<x<\infty, \\
v(0, t) & =1, & & 0 \leq t .
\end{array}
$$

The solution of (14) is given by

$$
v(x, t)=\operatorname{erfc}\left(\frac{x}{2 \sqrt{t}}\right),
$$

where

$$
\operatorname{erfc}(z)=\frac{2}{\sqrt{\pi}} \int_{z}^{\infty} e^{-u^{2}} d u
$$


Let us solve Eqs. (14) by the backward finite difference approximation. By choosing the mesh ratio $\Delta t /(\Delta x)^{2}=1$, and denoting $v(i \Delta x, j \Delta t)$ by $v_{i, i}$, one obtains the following "infinite" system of equations:

$$
\begin{aligned}
& \begin{array}{ll}
v_{1, i} & =1,
\end{array} \\
& -v_{1, i}+3 v_{2, i}-v_{3, i}=v_{2, j-1} \text {, } \\
& -v_{2, i}+3 v_{3, i}-v_{4, i}=v_{3, i-1}, \\
& -v_{3, i}+3 v_{4, i}-v_{5, i}=v_{4, i-1},
\end{aligned}
$$

The left carry-over factors are

$$
c_{2}=0, \quad c_{i+1}=1 /\left(3-c_{i}\right) .
$$

This is shown in the fourth row of Table 6 . Notice that $c_{i}$ approaches a constant value as $i$ increases. In fact, the difference equation (17) has a closed form solution (see [4]).

$$
c_{i+2}=\frac{\sinh \alpha i}{\sinh \alpha(i+1)}, \text { where } e^{\alpha}=(3+\sqrt{5}) / 2 .
$$

Hence the asymptotic value of $c_{i}$ as $i \rightarrow \infty$ is

$$
c_{\infty}=e^{-\alpha}=(3-\sqrt{5}) / 2=0.38196 \cdots .
$$

Now, let us determine the right carry-over factors. We can assume that $v(\infty, t)=0$. Then we have

$$
c_{\infty}^{\prime}=0, \quad c_{i-1}^{\prime}=1 /\left(3-c_{i}^{\prime}\right) .
$$

This is a difference equation similar to Eq. (17). According to the asymptotic behavior discussed above, we conclude that

$$
c_{1}^{\prime}=c_{2}^{\prime}=\cdots=c_{i}^{\prime}=(3-\sqrt{5}) / 2=0.38196 \cdots,
$$

as long as $i$ is finite. This is listed in the second row of Table 6 . The third row then gives the weight factors $w_{i}$. All the numbers in Table 6 are rounded off to three digits after the decimal point.

We remark that the asymptotic solution $c_{\infty}$ of Eq. (17) is independent of the initial value $c_{2}$. Hence, the assumption that $c_{\infty}^{\prime}=0$ in Eq. (20) is immaterial.

Since $v_{1,0}=1$, and $v_{i, 0}=0, i \neq 1$, the solution for the first step $v_{i, 1}$ is readily obtained by using the factors $c_{i}^{\prime}$ alone. This is shown in the fifth row. The solutions for the second and third steps are shown respectively in the ninth and thirteenth rows. In the eighth and twelfth rows, we have assumed that $C_{8,2}$ and $C_{8,3}$ are small and negligible. For comparison, we list the solution $v(i \Delta x, 3 \Delta t)$ obtained from Eq. (15) in the last row. It should be noticed that while $v(i \Delta x, 3 \Delta t)$ are the exact solution of Eqs. (14), $v_{i, 3}$ are the "exact" solutions (with \pm 1 round-off error in the last digit) of Eqs. (16).

5. Conclusions. The method described here is very convenient and efficient for most physical problems which have the system of equations in the form of Eqs. (4). The method has symmetry in the procedure of ealculation and has flexibility in that one can eliminate unnecessary computations. For instance, if we want to determine a particular $x_{i}$ only, say $x_{k}$, we do not need to find all or part of $x_{i}$ before we know the value of $x_{k}$. We can determine $x_{k}$ independently. 
TABLE 6. Solutions of Eqs. (16)

\begin{tabular}{|c|c|c|c|c|c|c|c|c|c|c|c|c|c|c|}
\hline (1) & $i$ & $=$ & & 2 & & 3 & & 4 & 5 & & 6 & 7 & & $8 \cdots$ \\
\hline (2) & $c_{i}^{\prime}$ & .382 & $\rightarrow$ & .382 & $\rightarrow$ & .382 & $\rightarrow$ & $.382 \rightarrow$ & .382 & $\rightarrow$ & $.382 \rightarrow$ & 382 & $\rightarrow$ & $.382 \ldots$ \\
\hline (3) & $w_{i}$ & $=1.000$ & & .382 & & .438 & & .446 & .447 & & .448 & .448 & & .448 \\
\hline (4) & $c_{i}$ & $=$ & $\leftarrow$ & 0 & $\leftarrow$ & .333 & $\leftarrow$ & $.375 \leftarrow$ & .381 & $\leftarrow$ & $.382 \leftarrow$ & .382 & $\leftarrow$ & $.382 \cdots$ \\
\hline (5) & $v_{i, 1}$ & $=1.000$ & & .382 & & .146 & & .056 & .021 & & .008 & .003 & & $.001 \cdots$ \\
\hline (6) & $C_{i, 2}^{\prime}$ & $=$ & & .382 & & .202 & & .102 & .049 & & .022 & .010 & & $.004 \cdots$ \\
\hline (7) & $W_{i, 2}^{i, 2}$ & $=1.000$ & & .146 & & .064 & & .025 & .009 & & .004 & .001 & & $.000 \ldots$ \\
\hline (8) & $C_{i, 2}$ & $=0$ & & .025 & & .011 & & .004 & .002 & & .000 & .000 & & $-\cdots$ \\
\hline$(9)$ & $v_{i, 2}$ & $=1.000$ & & .553 & & .277 & & .131 & .060 & & .026 & .011 & & $.004 \cdots$ \\
\hline (10) & $C_{i, 3}^{\prime}$ & $=$ & & .382 & & .226 & & .133 & .073 & & .038 & .019 & & .009 . \\
\hline (11) & $W_{\imath, 3}$ & $=1.000$ & & .211 & & .121 & & .058 & .027 & & .012 & .005 & & $.002 \cdots$ \\
\hline (12) & $C_{i, 3}$ & $=0$ & & .049 & & .026 & & .012 & .005 & & .002 & .001 & & $-\cdots$ \\
\hline (13) & $v_{i, 3}$ & $=1.000$ & & .642 & & .373 & & .203 & .105 & & .052 & .025 & & $.011 \cdots$ \\
\hline$v(i$ & $3 \Delta t)=$ & $=1.000$ & & .683 & & .414 & & .220 & .102 & & .040 & .014 & & $.004 \cdots$ \\
\hline
\end{tabular}

When the carry-over factor is infinite, a modified method is offered. This, perhaps, may complicate the programming for use in an electronic computer. However, this is rarely needed in ordinary physical problems.

Theoretically, this method is applicable regardless of the magnitude of the carryover factors. In numerical calculations, however, a large carry-over factor can cause serious round-off errors in the solution. Another "modified" method could be devised to prevent the large factors if one followed the classical method of selecting the largest pivot.

\section{APPENDix}

We shall discuss briefly the method described in section 2 in matrix notation and compare it with the traditional method of triangular decomposition. Let us write Eqs. (4) as

$$
A \mathrm{x}=\mathrm{d} .
$$

According to our method, the matrix $A$ is first decomposed into a lower triangular matrix $L$ and an unit upper triangular matrix $C$, namely,

$$
A=L C
$$

where

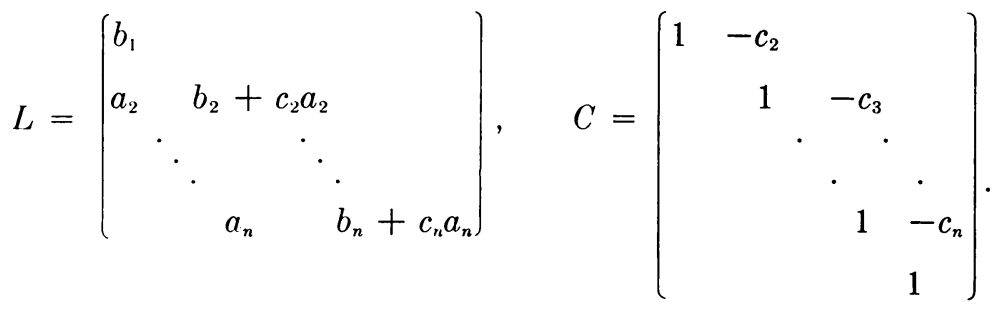


$c_{i}$ are defined in Eqs. (5). Next, in the same manner, we decompose $A$ into an upper triangular matrix $U$ and an unit lower triangular matrix $C^{\prime}$, namely,

$$
A=U C^{\prime}
$$

where

$$
U=\left(\begin{array}{ccc}
b_{1}+c_{1}^{\prime} a_{1}^{\prime} & a_{1}^{\prime} & \\
\ddots & \ddots & \\
b_{n-1}+c_{n-1}^{\prime} a_{n-1}^{\prime} & a_{n-1}^{\prime} \\
& & b_{n}
\end{array}\right), \quad C^{\prime}=\left(\begin{array}{ccccc}
1 & & & & \\
-c_{1}^{\prime} & 1 & & & \\
& \cdot & \cdot & & \\
& & -c_{n-2}^{\prime} & 1 & \\
& & & -c_{n-1}^{\prime} & 1
\end{array}\right) .
$$

$c_{i}^{\prime}$ are defined in Eqs. (8). If we define the diagonal matrix $W$ by

$$
W^{-1}=\left(\begin{array}{cccc}
w_{1} & & & \\
& w_{2} & & \\
& \ddots & \\
& & w_{n}
\end{array}\right)
$$

where $w_{i}$ are expressed by Eqs. (9), we have, finally

$$
A=L+U-W \text {. }
$$

Equation (24) with Eqs. (22) and (23) give

$$
\begin{aligned}
W & =L+U-A \\
& =A C^{-1}+A C^{\prime-1}-A \\
& =A\left(C^{-1}+C^{\prime-1}-I\right) .
\end{aligned}
$$

Hence

$$
A^{-1}=\left(C^{-1}+C^{-1}-I\right) W^{-1}
$$

and the solution of Eq. (21) becomes

$$
\mathbf{x}=\left(C^{-1}+C^{-1}-I\right)\left(W^{-1} \mathbf{d}\right) .
$$

This is the method described in section 2 (see Table 4).

The traditional method is equivalent to decomposing $A$ into a lower triangular matrix $L$ and an unit upper triangular matrix $C$ as shown in Eq. (22), and then decomposing $L$ into a diagonal matrix $D$ and an unit lower triangular matrix $C^{*}$. In other words,

$$
A=L C=D C^{*} C
$$

where

$$
D=\left(\begin{array}{cc}
b_{1} & \\
& b_{2}+c_{2} a_{2} \\
& \ddots \\
b_{n}+c_{n} a_{n}
\end{array}\right), \quad C^{*}=\left(\begin{array}{cccc}
1 & & \\
\frac{a_{n}}{b_{2}+c_{2} a_{2}} & 1 & & \\
& \cdot & \cdot \\
& & \frac{a_{n}}{b_{n}+c_{n} a_{n}} & 1
\end{array}\right) .
$$


Hence

$$
A^{-1}=C^{-1} C^{*-1} D^{-1}
$$

and the solution of $(21)$ is

$$
\mathbf{x}=C^{-1} C^{*-1}\left(D^{-1} \mathrm{~d}\right)
$$

In the traditional method, one can treat the elements of $D$ as weight factors and the codiagonal elements of $C$ and $C^{*}$ as carry-over factors. The procedure of calculation would be slightly different from our method. If one wanted to determine a particular element of $\mathbf{x}$ only, one has to invert the whole matrix $C^{*}$ and part of the matrix $C$ by Eq. (27), whereas by Eq. (25) only parts of matrices $C$ and $C^{\prime}$ need to be inverted.

Should one of the diagonal elements of the matrix $L$ vanish, the corresponding codiagonal elements of $C$ and $C^{*}$ become infinite and the methods represented by Eqs. (25) and (27) fail. Matrix notation for the method using modified carry-over factors are omitted here. However, if one follows the method described in section 3 and writes the method in matrix notation, one will find the modified carry-over factor placed in the third diagonal of $C$ (or $C^{\prime}$ ) with two zeros in the co-diagonal adjacent to it. There is a corresponding non-zero element in the third diagonal of $L$ (or $U$ ).

Acknowledgement. The author would like to thank Professor Bruce A. Chartres for his valuable comments on this paper.

\section{REFERENCES}

1 G. E. Forsythe and W. R. Wasow, Finite-difference methods for partial differential equations, John Wiley and Sons, Inc., Publishers, New York, London, 1960, p. 104

2. J. H. Wilkinson, Calculation of the eigenvectors of a symmetric tridiagonal matrix by inverse iteration, Numerische Mathematik 4, pp. 368-376 (1962)

3. R. Bellman, Introduction to malrix analysis, McGraw-Hill Book Co., Inc., New York, 1960

4. F. B. Hildebrand, Methods of applied mathematics, Prentice-Hall, Inc., 1954, p. 358 